\title{
GFP Labeling and Hepatic Differentiation Potential of Human Placenta-Derived Mesenchymal Stem Cells
}

\author{
Jiong $\mathrm{Yu}^{\mathrm{a}}$ Xiaoru Su ${ }^{\mathrm{a}}$ Chengxing Zhu ${ }^{\mathrm{a}}$ Qiaoling Pan ${ }^{\mathrm{a}} \quad$ Jinfeng Yang ${ }^{\mathrm{a}}$ Jing $\mathrm{Ma}^{\mathrm{a}}$ \\ Leyao Shen ${ }^{b}$ Hongcui Cao ${ }^{a}$ Lanjuan Lia \\ aThe State Key Laboratory for Diagnosis and Treatment of Infectious Diseases, First Affiliated Hospital, \\ College of Medicine, Zhejiang University; Collaborative Innovation Center for Diagnosis and Treatment \\ of Infectious Diseases, Hangzhou City, China, 'Biology Department, Vassar College, Poughkeepsie, New \\ York, USA
}

\section{Key Words}

Placenta-derived mesenchymal stem cell - Green fluorescent protein - Differentiation • Hepatocyte

\begin{abstract}
Background: Stem cell-based therapy in liver diseases has received increasing interest over the past decade, but direct evidence of the homing and implantation of transplanted cells is conflicting. Reliable labeling and tracking techniques are essential but lacking. The purpose of this study was to establish human placenta-derived mesenchymal stem cells (hPMSCs) expressing green fluorescent protein (GFP) and to assay their hepatic functional differentiation in vitro. Methods: The GFP gene was transduced into hPMSCs using a lentivirus to establish $\mathrm{GFP}^{+}$hPMSCs. GFP' ${ }^{+}$hPMSCs were analyzed for their phenotypic profile, viability and adipogenic, osteogenic and hepatic differentiation. The derived $\mathrm{GFP}^{+}$hepatocyte-like cells were evaluated for their metabolic, synthetic and secretory functions, respectively. Results: GFP $^{+}$hPMSCs expressed high levels of HLA I, CD13, CD105, CD73, CD90, CD44 and CD29, but were negative for HLA II, CD45, CD31, CD34, CD133, CD271 and CD79. They possessed adipogenic, osteogenic and hepatic differentiation potential. Hepatocyte-like cells derived from GFP' hPMSCs showed typical hepatic phenotypes. Conclusions: GFP gene transduction has no adverse influences on the cellular or biochemical properties of hPMSCs or markers. GFP gene transduction using lentiviral vectors is a reliable labeling and tracking method. GFP ${ }^{+}$ hPMSCs can therefore serve as a tool to investigate the mechanisms of MSC-based therapy, including hepatic disease therapy.
\end{abstract}

Hongcui Cao, M.D.

and Lanjuan Li, M.D.

KARGER 125
State Key Laboratory for Diagnosis and Treatment of Infectious Diseases,

The First Affiliated Hospital, School of Medicine, Zhejiang University

79 Qingchun Road, Hangzhou, 310003 (China)

Tel.+86-571-87236451,E-Mail hccao@zju.edu.cn and, ljli@zju.edu.cn 


\section{Cellular Physiology Cell Physiol Biochem 2015;35:2299-2308 \begin{tabular}{l|l} 
and Biochemistry $\begin{array}{l}\text { DOI 10.1159/000374033 } \\
\text { Published online: April 13, } 2015\end{array}$ & $\begin{array}{l}\text { C 2015 S. Karger AG, Basel } \\
\text { www.karger.com/cpb }\end{array}$ \\
\hline
\end{tabular} \\ Yu et al.: GFP Labeling Human Mesenchymal Stem Cells}

\section{Introduction}

Human placenta-derived mesenchymal stem cells (hPMSCs) have recently been proven as attractive candidates for cell-based therapy due to their practical and ethical advantages: they are easy to collect, have a high proliferation rate for expansion in vitro and they are able to differentiate into multiple lineages, including chondrocytes, osteoblasts and adipocytes [1]. Increasing evidence of the hepatic capabilities of hPMSCs both in vitro and in vivo shows that hPMSCs may be a preeminent transplantable source of hepatic progenitors that do not require invasive surgery. Some studies have attempted to demonstrate hPMSCs homing to the liver and differentiating into normal hepatocytes; hPMSCs can also potentially activate and support the differentiation and proliferation of liver progenitor cells. Differentiated hepatic cells are capable of secreting multiple cytokines and growth factors to reduce inflammation and angiogenesis and accelerate restoration of the liver [2-4]. However, there is no convincing evidence that the biological characteristics of hPMSCs, including homing, differentiation and secretion, are responsible for their therapeutic effects in vivo. This results from the difficulty in tracking mesenchymal stem cells (MSCs) after transplantation due to the lack of reliable MSC-specific markers in vivo. Nowadays, MSCs can be stably labeled or stained in vivo using numerous new methods involving 5-bromo-2'-deoxyuridine (BrdU), magnetic nanoparticles, 18-fludeoxyglucose (18FDG) and green fluorescent protein (GFP) [5-9].

The present study aimed to assess the GFP genetic modification of hPMSCs, in terms of transduction efficiency and durability of transgene expression, using a lentiviral vector. In addition, it examined the effect of lentiviral vector transduction on hPMSC (GFP' ${ }^{+}$PMSC) morphology, phenotypic profiles, viability and the capacity for adipogenic, osteogenic and hepatic differentiation, compared with unlabeled hPMSCs.

\section{Materials and Methods}

\section{GFP transduction}

All protocols for human tissue and cell handling were approved by the Research Ethics Committee of the First Affiliated Hospital, School of Medicine, Zhejiang University, Zhejiang, China (Reference no. 2013272). hPMSCs were collected from fresh human placentas and prepared as described previously [3]. The day before transduction, hPMSCs (passage 3) were cultured in a 6-well plate at a density of $1 \times 10^{4} / \mathrm{cm}^{2}$ in special medium (MesenCult ${ }^{\circledR}$ Human Basal Medium plus MesenCult Human Supplement; STEMCELL Technologies Inc., Vancouver, $\mathrm{BC}$, Canada). On the day of transduction, the medium was aspirated, and the cells were washed once with PBS. Then, $2 \mathrm{ml}$ of new culture medium containing $5 \mu \mathrm{g} / \mathrm{ml}$ polybrene were added to each well. Next, $100 \mu \mathrm{l}$ of $1 \times 10^{9} \mathrm{TU} / \mathrm{ml}$ Lentivirus- 5 negative control (LV-5NC) recombinant lentivirus (GenePharma, Shanghai, China) were added, and the media were mixed gently. The cells were incubated overnight at $37^{\circ} \mathrm{C}$ in a humidified atmosphere containing $5 \%(\mathrm{v} / \mathrm{v}) \mathrm{CO}_{2}$. The next day, morphologic changes and GFP expression were observed using a fluorescence phase contrast microscope (IX2-UCB-2; Olympus, Tokyo, Japan). The medium was then replaced with fresh medium containing $10 \mu \mathrm{g} / \mathrm{ml}$ puromycin (SigmaAldrich, St. Louis, MO, USA). Upon reaching 80-90\% confluence, adherent cells were trypsinized using $0.25 \%(\mathrm{w} / \mathrm{v}$ ) trypsin/EDTA (Invitrogen, Carlsbad, CA, USA) and re-plated at a 1:3 dilution.

\section{GFP expression verification}

GFP-transduced hPMSCs (GFP+ hPMSCs) at passages 5 and 20 were washed with PBS twice, and 5 $\times 10^{6}$ cells were resuspended to $500 \mu \mathrm{L}$ of T-PER ${ }^{\circledR}$ Tissue Protein Extraction Reagent (Pierce, Rockford, IL, USA), supplemented with $1 \mathrm{mM}$ phenylmethanesulfonyl fluoride (Pierce) and $5 \mathrm{mM}$ ethylene diamine tetraacetic acid (EDTA; Sigma-Aldrich), according to the manufacturer's instructions. The mixture was centrifuged at $20000 \times \mathrm{g}$ for $15 \mathrm{~min}$ and the protein was quantitated using a BCA ${ }^{\mathrm{TM}}$ Protein Assay Kit (Pierce). The proteins were separated on $12 \%$ polyacrylamide gels and transferred to polyvinylidene fluoride membranes (Millipore, Bedford, MA, USA). The blots were incubated for $1 \mathrm{~h}$ in Tris-buffered saline (TBS; 20 $\mathrm{mM}$ Tris- $\mathrm{HCl}$ and $140 \mathrm{mM} \mathrm{NaCl}, \mathrm{pH}$ 7.5) with $0.05 \%$ Tween-20 and 5\% skim milk at room temperature, then 


\section{Cellular Physiology Cell Physiol Biochem 2015;35:2299-2308 \begin{tabular}{ll|l} 
and Biochemistry & $\begin{array}{l}\text { DOI: 10.1159/000374033 } \\
\text { Published online: April 13, } 2015\end{array}$ & $\begin{array}{l}\text { O 2015 S. Karger AG, Basel } \\
\text { www.karger.com/cpb }\end{array}$ \\
\cline { 2 - 3 }
\end{tabular} \\ Yu et al.: GFP Labeling Human Mesenchymal Stem Cells}

incubated with anti-GFP antibodies (Abcam, Cambridge, UK) diluted 1:2000 overnight at $4{ }^{\circ} \mathrm{C}$. The blots were incubated with horseradish peroxidase-conjugated secondary antibodies (Abcam) diluted 1:3000 for $1 \mathrm{~h}$ at room temperature, and then developed using electrochemiluminescence reagents (Pierce). Recombinant Aequorea victoria GFP (Sino Biological Inc., Beijing, China) served as a positive control.

\section{Surface antigen expression}

$\mathrm{GFP}^{+}$hPMSCs (passages 2-5) were washed with PBS containing $0.5 \%(\mathrm{w} / \mathrm{v}$ ) bovine serum albumin (BSA) and adjusted to $1 \times 10^{6}$ cells $/ \mathrm{ml}$. They were then incubated in the dark with allophycocyanin-labeled anti-human antibodies against human leukocyte antigen class I (HLA I), HLA II, cluster of differentiation (CD)13, CD27, CD29, CD31, CD34, CD44, CD45, CD73, CD90, CD105 (eBioscience Inc., San Diego, CA, USA), CD133 (Miltenyi Biotec Inc., San Diego, CA, USA), CD79b and CD271 (Becton, Dickinson and Co., Franklin Lakes, NJ, USA) for $30 \mathrm{~min}$. The cells were next washed twice with PBS. Two-color flow cytometry was conducted using a Cytomics FC 500 MPL flow cytometer (Beckman Coulter, Brea, CA, USA), and the data were analyzed using MXP software.

\section{Cell viability and population doubling time assay}

Cell viability was determined using the 3-(4,5-dimethylthiazol-2-yl)-2,5-diphenyltetrazolium bromide (MTT) assay. Each group of cells was seeded at $3 \times 10^{3} / \mathrm{cm}^{2}$ in 96 -well plates (Nunclon ${ }^{\text {TM }}$ Delta Surface, Roskilde, Denmark). Next, $20 \mu \mathrm{l}$ of MTT reagent (Sigma-Aldrich) was added to each well. The resulting formazan complex was dissolved in $150 \mu \mathrm{l}$ of dimethyl sulfoxide (Sigma-Aldrich), and the absorbance at $490 \mathrm{~nm}$ was measured using a spectrophotometer (Multimode Detector DTX880; Beckman Coulter). To calculate the population doubling time (PDT), $3 \times 10^{3}$ cells $/ \mathrm{cm}^{2}$ were seeded in 24-well plates (Nunclon ${ }^{\mathrm{TM}}$ Delta Surface). The doubling time of the $\mathrm{GFP}^{+}$hPMSCs and control cells were calculated according to the formula: PDT $=\mathrm{t} \log 2 /\left(\log N_{\mathrm{t}}-\log N_{0}\right)$, where $t$ is the time to confluence, $N_{\mathrm{t}}$ is the number of cells at the end of the growth period, and $\mathrm{N}_{0}$ is the initial number of cells [10].

\section{Adipogenic and osteogenic differentiation}

$\mathrm{GFP}^{+}$hPMSCs (passages 2-5) were plated at a density of $1 \times 10^{3} / \mathrm{cm}^{2}$ on 6-well multidishes (Nunclon ${ }^{\mathrm{TM}}$ Delta Surface) and cultured in adipogenic or osteogenic medium (OriCell ${ }^{\mathrm{TM}}$ hMSC Adipogenic and Osteogenic Differentiation Medium; Cyagen Biosciences, Guangzhou, China) according to the manufacturer's protocol. Four weeks later, adipogenesis was confirmed by staining with oil red 0 (Sinopharm Chemical Reagent Co., Ltd., Shanghai, China), and osteogenic differentiation was detected by staining with alizarin red S (Genmed Scientifics Inc. USA, Shanghai, China).

\section{Hepatic differentiation}

Hepatogenic induction was conducted as follows. GFP ${ }^{+}$hPMSCs $\left(1 \times 10^{3} / \mathrm{cm}^{2}\right.$, passages $\left.2-5\right)$ were plated in T25 culture flasks (Nunc flasks with a filter cap; Nunclon ${ }^{\mathrm{TM}}$ Delta Surface) in LG-DMEM (Life Technologies Inc., Gibco, Grand Island, NY, USA) containing 10\% (v/v) FBS (Gibco). After 2 days, the medium was changed to Iscove's modified Dulbecco's medium (IMDM) supplemented with $20 \mathrm{ng} / \mathrm{ml}$ epidermal growth factor (Sigma-Aldrich) and $10 \mathrm{ng} / \mathrm{ml}$ basic fibroblast growth factor (FGF; Sigma-Aldrich) for 2 days. The medium was subsequently changed to IMDM supplemented with $20 \mathrm{ng} / \mathrm{ml}$ hepatocyte growth factor (Sigma-Aldrich), $10 \mathrm{ng} / \mathrm{ml} \mathrm{FGF,} 0.61 \mathrm{~g} / \mathrm{l}$ niacin amide and 1\% (v/v) insulin-transferrin-selenium (ITS) premix (Sigma-Aldrich) for 10 days. The medium was replaced every 3 days. Finally, the cells were cultured in IMDM with $20 \mathrm{ng} / \mathrm{ml}$ oncostatin M (Sigma-Aldrich), 1 mol/l dexamethasone (Sigma-Aldrich) and 1\% (v/v) ITS premix for 10 days.

\section{Immunocytochemistry}

Cells were fixed in $4 \%(\mathrm{v} / \mathrm{v})$ paraformaldehyde at room temperature for about 15 min, treated with $0.3 \%(\mathrm{v} / \mathrm{v})$ hydrogen peroxide and blocked in $5 \%(\mathrm{~m} / \mathrm{v})$ BSA, then incubated with primary antibodies against human cytokeratin 18 (CK18) (Abcam) diluted 1:400, CK19 (Abcam) diluted 1:400, human alphafetoprotein (AFP; Abcam) diluted 1:200, or human albumin (ALB; Abcam) diluted 1:500, according to the manufacturer's instructions. Then they were detected by incubation with horseradish peroxidase-conjugated anti-rabbit IgG antibodies (Abcam) diluted 1:1000, followed by diaminobenzidine tetrahydrochloride solution (DAB kit; Vector Labs, Burlingame, CA, USA).

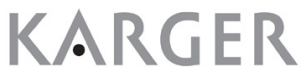




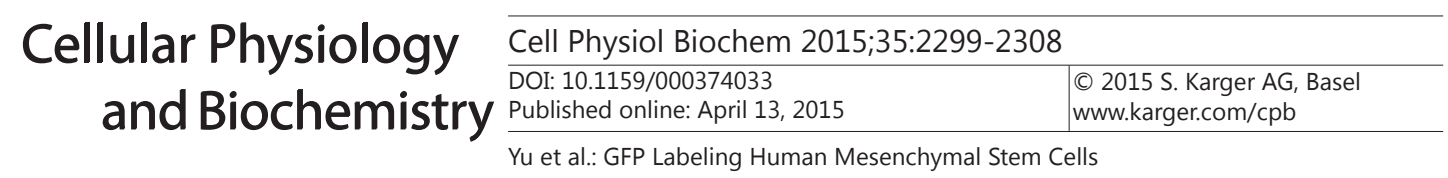

Table 1. Sequences of the primers used in this study

\begin{tabular}{|c|c|c|c|}
\hline Primer & Sequence & $\begin{array}{l}\text { Amplicon } \\
\text { size (bp) }\end{array}$ & Cycle \\
\hline \multirow[b]{2}{*}{ ALB } & 5'- CACAGTTGCAACTCTTCGTGAAAC-3' & \multirow{2}{*}{154} & \multirow{2}{*}{$94^{\circ} \mathrm{C} / 30 \mathrm{~s} ; 55^{\circ} \mathrm{C} / 30 \mathrm{~s} ; 72^{\circ} \mathrm{C} / 60 \mathrm{~s}$} \\
\hline & 5'- AGCAGTGCACATCACATCAACC-3' & & \\
\hline \multirow[b]{2}{*}{ AFP } & 5'- GCATGTGCAGTAATGAAAAAT-3' & \multirow{2}{*}{400} & \multirow{2}{*}{$94^{\circ} \mathrm{C} / 30 \mathrm{~s} ; 50^{\circ} \mathrm{C} / 30 \mathrm{~s} ; 72^{\circ} \mathrm{C} / 60 \mathrm{~s}$} \\
\hline & 5'- GAACAAAACTTGCCAAGAAGAT-3' & & \\
\hline \multirow{2}{*}{ CK18 } & 5'- CACCGTCGTCCGCAAAGCCT-3' & \multirow{2}{*}{269} & \multirow{2}{*}{$94^{\circ} \mathrm{C} / 60 \mathrm{~s} ; 58^{\circ} \mathrm{C} / 60 \mathrm{~s} ; 72^{\circ} \mathrm{C} / 120 \mathrm{~s}$} \\
\hline & 5'- CCTGCCAGACCCCCGGCTAT-3' & & \\
\hline \multirow[b]{2}{*}{ CK19 } & 5'-AGGTGGATTCCGCTCCGGGCA-3' & \multirow[b]{2}{*}{460} & \multirow{2}{*}{$94^{\circ} \mathrm{C} / 120 \mathrm{~s} ; 64^{\circ} \mathrm{C} / 120 \mathrm{~s} ; 72^{\circ} \mathrm{C} / 180 \mathrm{~s}$} \\
\hline & 5'- ATCTTCCTGTCCCTCGAGCA-3' & & \\
\hline \multirow{2}{*}{$\beta$-Actin } & 5'- GAGCGGGAAATCGTGCGTGACATT-3' & \multirow{2}{*}{234} & \multirow{2}{*}{$94^{\circ} \mathrm{C} / 60 \mathrm{~s} ; 58^{\circ} \mathrm{C} / 30 \mathrm{~s} ; 72^{\circ} \mathrm{C} / 60 \mathrm{~s}$} \\
\hline & 5'- GATGGAGTTGAAGGTAGTTTCGTG-3' & & \\
\hline
\end{tabular}

Reverse transcriptase-polymerase chain reaction (RT-PCR)

Total RNA was extracted from cells using TRIzol reagent (Invitrogen). The RNA was quantitated by measuring the absorbance at 260 and $280 \mathrm{~nm}$. cDNA was synthesized using oligo dT primers and the ImProm-II ${ }^{\mathrm{TM}}$ Reverse Transcription System (Promega, Madison, WI, USA) according to the manufacturer's instructions. The primer sequences are shown in Table 1.

Glycogen storage staining

Differentiated and control cells were subjected to oxidation and then stained using a periodic acidSchiff (PAS) staining kit (Genmed Scientifics Inc. USA).

Low-density lipoprotein (LDL) uptake assay

Differentiated and control cells were labeled with $10 \mu \mathrm{g} / \mathrm{ml}$ 1,19-dioctadecyl-3,3,39,39tetramethyllindocarbocyane-low-density lipoprotein (DiI-LDL; Molecular Probes, Invitrogen) for $24 \mathrm{~h}$ at $37^{\circ} \mathrm{C}$. The cells were then washed with PBS and evaluated using an IX81 fluorescence microscope (Olympus).

Urea assay

Differentiated and control cells were cultured for $24 \mathrm{~h}$ in HepatoZYME-SFM (serum-free, no phenol red; Gibco) supplemented with $0.9 \mathrm{mmol} / \mathrm{l}$ ammonium chloride (Bio Basic Inc., Amherst, NY, USA). The supernatants were collected and the urea levels were assayed using a Urea Assay Kit (BioAssay Systems, Hayward, CA, USA) by measuring the absorbance at $430 \mathrm{~nm}$ with a spectrophotometer (Multimode Detector DTX880; Beckman Coulter).

Cytochrome P450 activity

Cells were cultured with medium containing $10 \mu \mathrm{M}$ rifampicin for $48 \mathrm{~h}$, after which the medium was changed to IMDM containing a luminogenic CYP substrate (50 $\mu \mathrm{M}$ luciferin-PFBE, 1:40 dilution) for $24 \mathrm{~h}$. Intracellular CYP450 activity was assessed by assaying luciferin production (P450-Glo ${ }^{\mathrm{TM}} \mathrm{CYP} 3 \mathrm{~A} 4$ assay with luciferin-PFBE; Promega).

\section{Statistical analysis}

The data are presented as means \pm standard deviation (SD). Statistical analyses for cell viability, immunophenotype, urea synthesis, CYP-450 enzymatic activity and secretion were performed using $t$-tests. Values of $p<0.05$ were considered statistically significant $\left({ }^{*} p<0.05,{ }^{*} p<0.01\right)$. The data were analyzed using SPSS version 16.0 (IBM Corp., Armonk, NY, USA).

\section{Results}

Morphology and phenotypic profile

We transduced hPMSCs with a lentiviral vector encoding GFP and assessed successful lentiviral transduction in viable cultured MSCs directly using a fluorescence phase contrast microscope to detect GFP expression (Fig. 1-I). At a multiplicity of infection (MOI) of 100, KARGER 


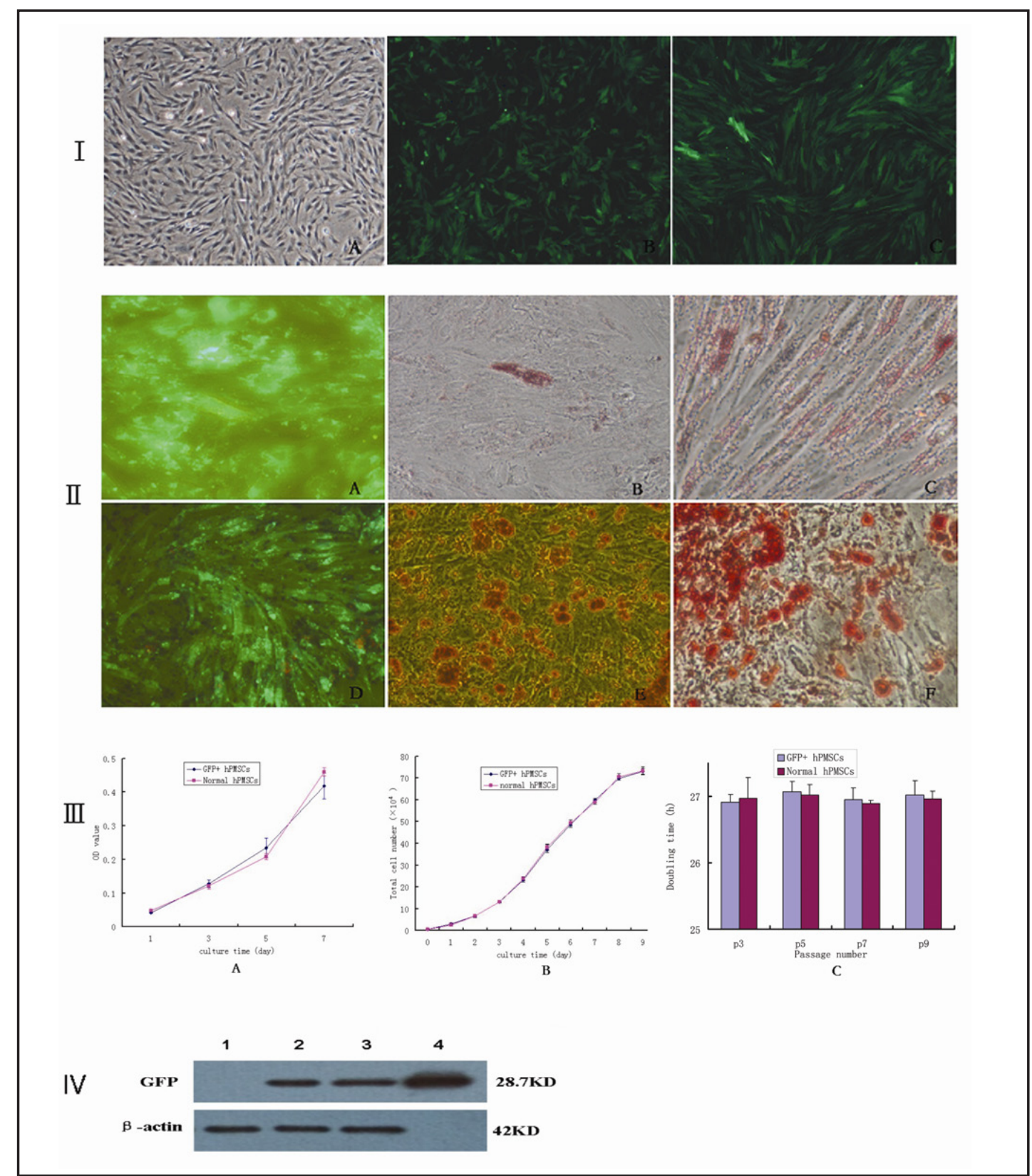

Fig. 1. (I) hPMSCs morphology (40x). (A) hPMSCs without lentiviral transduction. (B) hPMSCs with lentiviral transduction after 3 days. (C) Passage 20 after lentiviral transduction. (II) Adipogenic (A-C) and osteogenic (D-F) differentiation of GFP ${ }^{+}$hPMSCs. (A and B) GFP ${ }^{+}$hPMSCs that underwent adipogenic differentiation (accumulation of lipid droplets is shown by red staining) before and after staining (100×). (C) hPMSCs that underwent adipogenic differentiation after staining (200x). (D and E) GFP ${ }^{+}$PPMSCs that underwent osteogenic differentiation (forming bone nodules) before and after staining (100x). (F) hPMSCs that underwent osteogenic differentiation after staining (200×). (III) GFP+ $\mathrm{hPMSC}$ viability and population doubling time compared with normal hPMSCs. (A) Viability of GFP' hPMSCs and non-transduced control hPMSCs. (B) Growth curves for GFP $\mathrm{GPMSCs}^{+}$and non-transduced control hPMSCs. (C) Population doubling time for $\mathrm{GFP}^{+}$hPMSCs at passages 3, 5, 7 and 9 compared with control hPMSCs. (IV) Western blot results. No GFP expression was found in normal hPMSCs (lane 1); GFP expression was found in $\mathrm{GFP}^{+} \mathrm{hPMSC}$ at passages 5 and 20 (lanes 2 and 3); recombinant $A$. victoria GFP served as a positive control (lane 4).

the vector yielded transduction efficiencies $>80 \%$. The stabilization of GFP expression at passages 5 and 20 was confirmed by Western blotting (Fig. 1-IV). 
Fig. 2. Surface antigen expression of $\mathrm{GFP}^{+} \mathrm{hP}-$ MSCs. Immunophenotype of $\mathrm{GFP}^{+}$hPMSCs determined by flow cytometry at passage 3 using labeled antibodies specific for the indicated human surface antigens or negative controls.
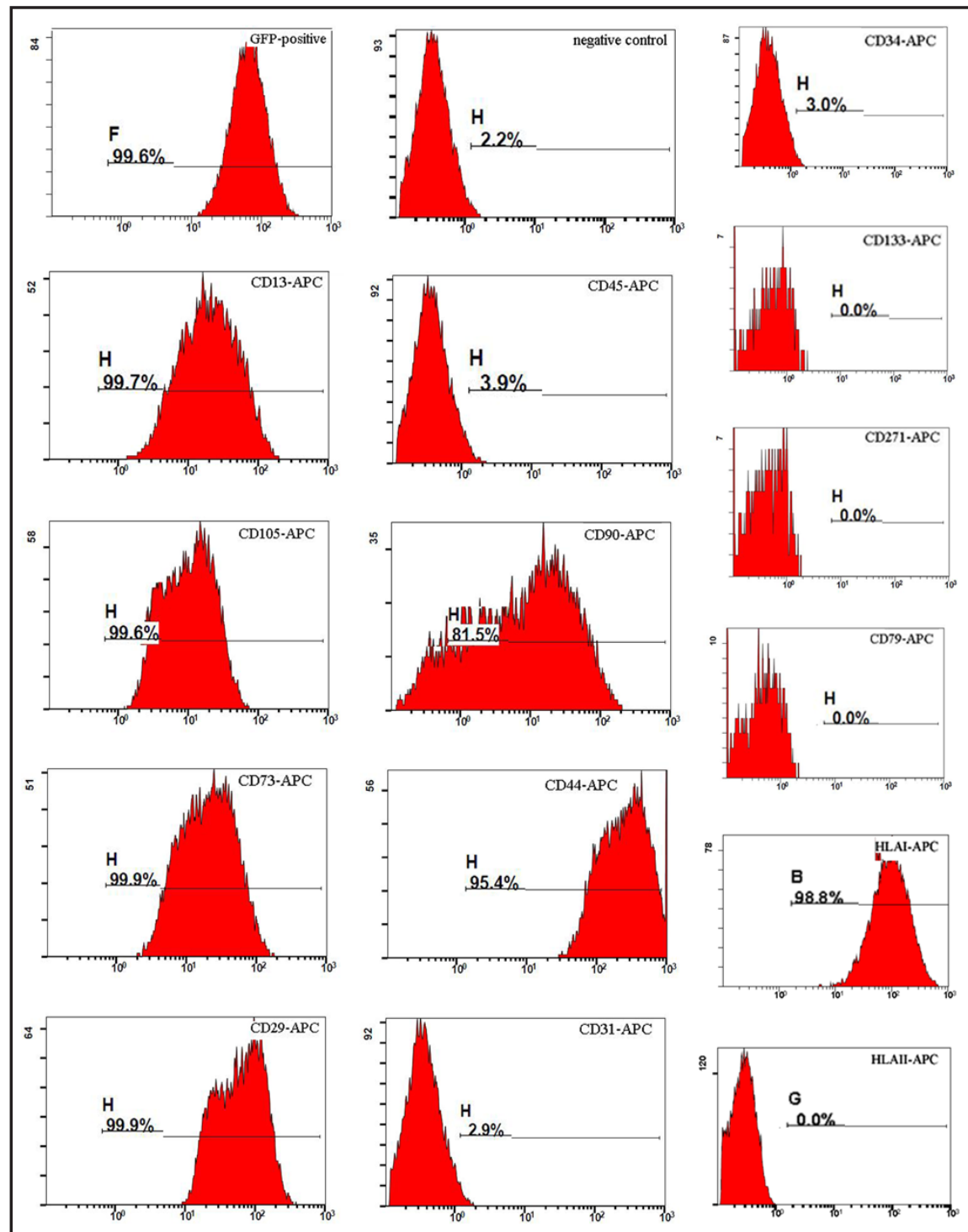

Table 2. Immunophenotypical comparison of $\mathrm{GFP}^{+}$hPMSCs and hPMSCs by flow cytometry

\begin{tabular}{lll}
\hline Immunophenotype & \multicolumn{2}{l}{ Expression rate (means \pm SD, n=3) } \\
& GFP+hPMSCs & hPMSCs \\
\hline HLA I & $98.87 \pm 0.35 \%$ & $97.53 \pm 0.35 \%$ \\
HLA II & $2.07 \pm 0.12 \%$ & $2.43 \pm 0.06 \%$ \\
CD45 & $3.93 \pm 0.06 \%$ & $4.97 \pm 0.15 \%$ \\
CD31 & $2.90 \pm 0 \%$ & $2.97 \pm 0.11 \%$ \\
CD34 & $3.0 \pm 0 \%$ & $2.93 \pm 0.06 \%$ \\
CD133 & $0 \%$ & $0 \%$ \\
CD271 & $0 \%$ & $0 \%$ \\
CD79 & $0 \%$ & $0 \%$ \\
CD13 & $99.63 \pm 0.25 \%$ & $98.63 \pm 0.06 \%$ \\
CD105 & $99.67 \pm 0.42 \%$ & $99.93 \pm 0.06 \%$ \\
CD73 & $99.93 \pm 0.06 \%$ & $99.83 \pm 0.12 \%$ \\
CD90 & $81.70 \pm 1.31 \%$ & $83.07 \pm 0.45 \%$ \\
CD44 & $95.43 \pm 0.31 \%$ & $99.27 \pm 0.55 \%$ \\
CD29 & $99.9 \pm 0 \%$ & $100 \pm 0 \%$ \\
\hline
\end{tabular}

The immunophenotype of the $\mathrm{GFP}^{+} \mathrm{hPMSC}$ s was analyzed by flow cytometry. The results indicate that the cells were negative for HLA II, CD45, CD31, CD34, CD133, CD271 and CD79, 
Fig. 3. (I) Hepatogenic differentiation of $\mathrm{GFP}^{+}$hPMSCs (100x). (A, D, G and J) Staining for the hepatic markers ALB, AFP, CK18 and CK19; a large number of cells were light brown in color. (B, E, H and $\mathrm{K}$ ) hPMSCs cultured in normal medium did not display positive staining. (C, F, I and L) C3A was used as a positive control. (II) RT-PCR analysis of hepatic-specific gene expression in hepatogenic differentiated $\mathrm{GFP}^{+} \mathrm{hP}$ MSCs. Lane 1, GFP+ hPMSCs cultured in normal growth medium; lane 2, $\mathrm{GFP}^{+}$ hPMSCs that underwent hepatic differentiation; lane 3 , human liver cancer tissue as a positive control. (III) Functional parameters of hepatocyte-like cells derived from $\mathrm{GFP}^{+}$hPMSCs. (A-C) Glycogen storage capacity (100×). (A) $\mathrm{GFP}^{+}$hPMSCs cultured in normal growth medium.
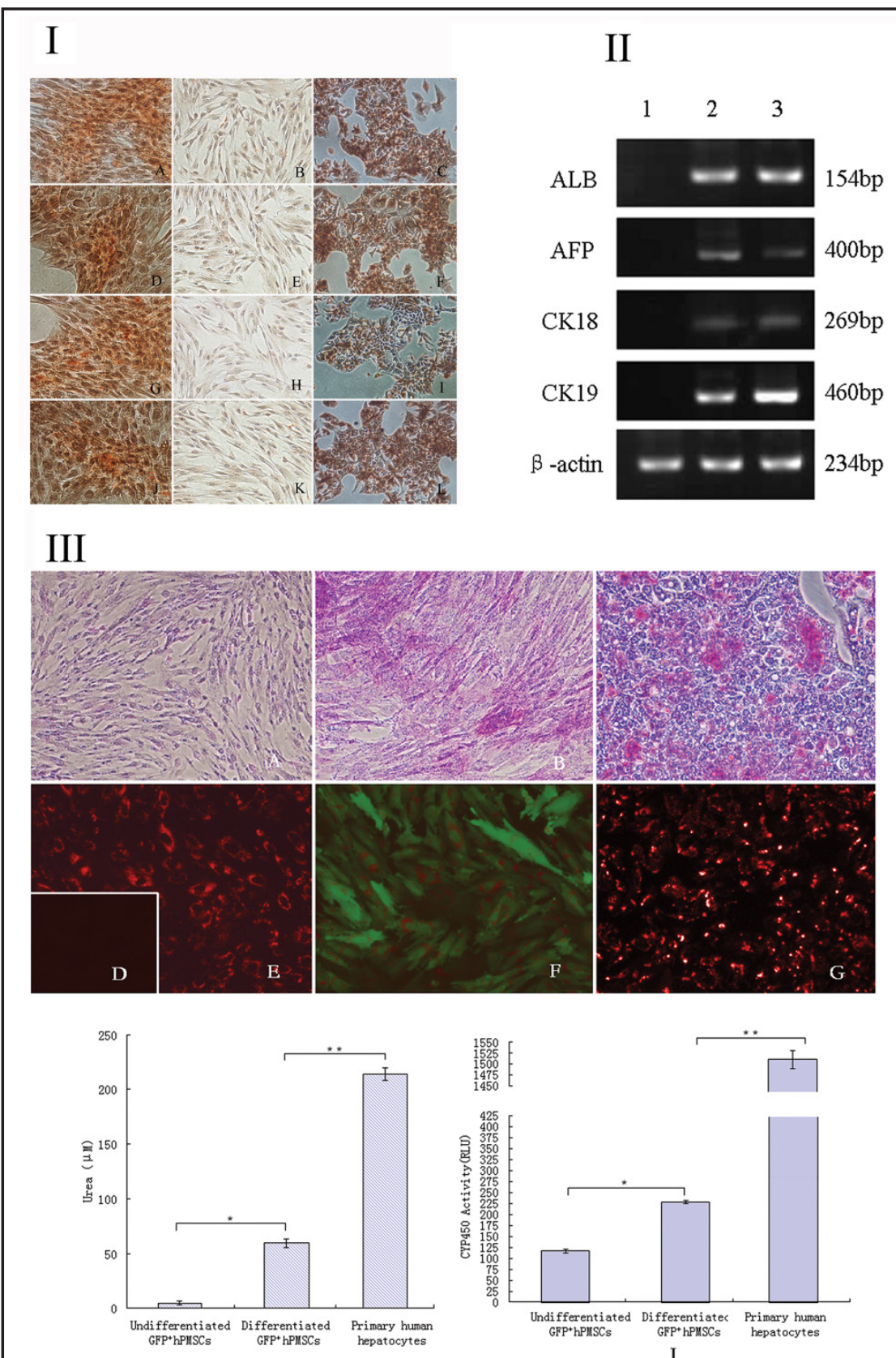

$\mathrm{H}$

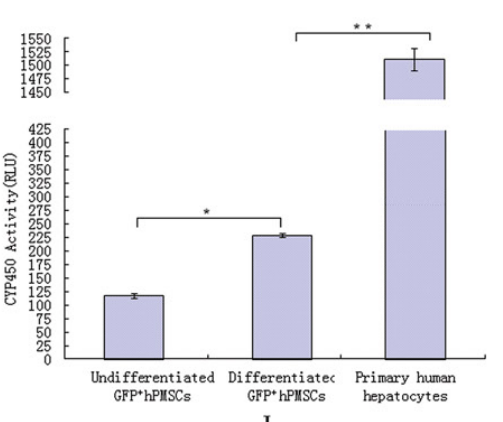

I

(B) $\mathrm{GFP}^{+}$hPMSCs cultured in hepatogenic differentiation medium for 28 days stained positively, revealing cytoplasmic glycogen accumulation. (C) C3A served as a positive control. (D-G) LDL uptake (100×). (D) GFP hPMSCs maintained in normal growth medium stained negatively. (E and F) LDL uptake was detected in hepatocyte-like cells 21 days after differentiation. (G) C3A served as a positive control. (H) Urea synthesis assay. When $\mathrm{GFP}^{+} \mathrm{hPMSC}$ were cultured in hepatogenic differentiation medium for 28 days, the urea levels in the culture supernatants were increased significantly. (I) CYP-450 enzymatic activity assay. After incubation in hepatogenic differentiation culture medium for 30 days, CYP-450 enzymatic activity was increased significantly. The data represent means $\pm \operatorname{SD}\left(\mathrm{n}=4 ;^{*} p<0.05,{ }^{* *} p<0.01\right)$.

but they expressed high levels of HLA I, CD13, CD105, CD73, CD90, CD44 and CD29. The data for passage 3 are shown in Figure 2. This is consistent with the general description of the phenotypic profile of classical hPMSCs (Table 2).

\section{KARGER}


Cell viability and PDT

After lentiviral vector transduction, cell viability was measured by an MTT assay (Fig. 1-IIIA). No significant adverse effects on cell viability on days 1, 3, 5 and 7 were apparent when $\mathrm{GFP}^{+} \mathrm{hPMSC}$ were compared with non-transduced control hPMSCs $\left(\mathrm{n}=3,{ }^{*} p>0.05\right)$. The growth curves of $\mathrm{GFP}^{+}$and control hPMSCs were both S-shaped (Fig. 1-IIIB). At generations $3,5,7$ and 9, the doubling time was $26.91 \pm 0.12,27.07 \pm 0.15,26.95 \pm 0.18$ and $27.01 \pm 0.22 \mathrm{~h}$, respectively, for $\mathrm{GFP}^{+} \mathrm{hPMSCs}$ (Fig. 1-IIIC); no significant adverse effects on doubling time were detected compared with non-transduced control hPMSCs $\left(\mathrm{n}=3,{ }^{*} p>0.05\right)$.

\section{Adipogenic and osteogenic differentiation}

Adipogenic differentiated $\mathrm{GFP}^{+}$hPMSCs formed intracytoplasmic lipid droplets and could be identified by oil red 0 staining. Osteogenic differentiated cells formed bone nodules and were positive on alizarin red S staining (Fig. 1-II).

\section{Hepatic differentiation}

The differentiation efficiency was confirmed by analyzing protein and mRNA expression. Immunocytochemistry revealed the presence of hepatic markers (ALB, AFP, CK18 and CK19) in $\mathrm{GFP}^{+}$hPMSCs subjected to the hepatogenic differentiation protocol, as well as in hepatocytes, but not in undifferentiated hPMSCs (Fig. 3-I). Similar results were obtained by RT-PCR (Fig. 3-II), which revealed the expression of ALB, CK-18 and CK-19 mRNA in differentiated cells.

Hepatocyte-like cells that had differentiated from $\mathrm{GFP}^{+}$hPMSCs exhibited typical hepatocyte features, including glycogen storage, LDL uptake, urea synthesis and CYP-450 enzymatic activity, which were absent in normal medium (Fig. 3-III).

\section{Discussion}

To further improve MSC-based treatment, certain crucial questions must be addressed. How can transplanted MSCs be engrafted and distributed to the target area? How can injected MSCs achieve their expected therapeutic effect? Cell labeling and tracking can help address these questions. BrdU, magnetic nanoparticles, 18FDG and GFP are markers commonly used to label and track stem cells. BrdU can be incorporated into DNA during cell replication because of its role as a thymidine analog. However, some research showed that BrdU labeling might be lost since it can be transferred to host cells [11]. Magnetic nanoparticles can also be used for cell labeling and can be tracked by magnetic resonance imaging in vivo. However, the label will be diluted as the MSCs divide, which is a major limitation for longterm tracking. Furthermore, macrophages can engulf dead MSCs containing nanoparticles, which will interfere with the tracking of labeled stem cells [12-14]. The in vivo application of 18FDG labeling has been limited to early biodistribution studies and requires positron emission tomography [7]. Therefore, BrdU, magnetic nanoparticles and 18FDG are not ideal biomarkers for studying hPMSCs.

GFP, first isolated from jellyfish, is a vital marker in mammalian cells [15]. Since GFP does not require any additional substrate for signal emission and can be scored directly by fluorescence microscopy, it has been widely used to track cells [16]. MSCs transfected with GFP can be tracked by fluorescence microscopy after cell transplantation. In our study, lentiviral vectors expressing GFP were integrated into the genome of hPMSCs, resulting in stable long-term GFP expression by passage 20. As expected, a dose-response effect was achieved in that the transfection efficiency increased as the MOI increased (the transduction efficiency was $>80 \%$ at an MOI of 100 ). We also found that lentiviral vector modification had no significant negative effects in hPMSCs compared with non-transduced control cells.

Besides the assessment of transduction efficiency and viability, we also examined the phenotypic profile and differentiation potential of MSCs modified with the lentiviral vector. hPMSCs transfected with lentivirus were positive for HLA I, CD13, CD105, CD73, CD90, 


\section{Cellular Physiology Cell Physiol Biochem 2015;35:2299-2308 \begin{tabular}{l|l} 
DoI: 10.1159/000374033 & \\
and Biochemistry Published online: April 13, 2015 & $\begin{array}{l}\text { 2015 S. Karger AG, Basel } \\
\text { www.karger.com/cpb }\end{array}$
\end{tabular}

CD44 and CD29 and negative for HLA II, CD45, CD31, CD34, CD133, CD271 and CD79, corroborating the results of previous studies [3]. MSCs are typically characterized by their demonstrated ability to differentiate into adipocytes and osteocytes [1]. In our study, GFP ${ }^{+}$ hPMSCs differentiated successfully into adipocytes and osteocytes. In addition, the GFP ${ }^{+}$ hPMSCs were able to differentiate into hepatocyte-like cells and acquired the ability to take up LDL, clear ammonia and store glycogen. Based on these data, it seems unlikely that differentiation pathways are affected by lentiviral modification. Indeed, some researchers observed that lentiviral vector transduction did not negatively affect the osteogenic or adipogenic differentiation of MSCs $[17,18]$.

In conclusion, we report that transduction of the GFP gene into MSCs using a lentiviral vector is a reliable labeling and tracking method, and that lentiviral vectors are valuable gene delivery tools for the lentivirus-mediated genetic modification of MSCs for MSC-based liver disorder therapies.

\section{Abbreviations}

GFP (green fluorescent protein); MSC (mesenchymal stem cell); hPMSC (human placenta-derived mesenchymal stem cell); MOI (multiplicity of infection); BrdU (5-bromo-2'deoxyuridine); EDTA (ethylene diamine tetraacetic acid); BSA (bovine serum albumin); MTT (3-(4,5-dimethylthiazol-2-yl)-2,5-diphenyltetrazolium bromide); FGF (fibroblast growth factor); IMDM (Iscove's modified Dulbecco's medium); ITS (insulin-transferrin-selenium); CK18 (cytokeratin 18); ALB (albumin); RT-PCR (reverse transcriptase-polymerase chain reaction); PAS (periodic acid-Schiff); LDL (low-density lipoprotein); CYP450 (cytochrome P450); SD (standard deviation); 18FDG (18-fludeoxyglucose); DiI-LDL (1,19-dioctadecyl3,3,39,39-tetramethylllindocarbocyane-low-density lipoprotein); HLA (human leukocyte antigen class); AFP (human alpha-fetoprotein); PDT (population doubling time).

\section{Acknowledgments}

This study was supported by the National Natural Science Foundation of China (No. 81471794), Chinese High Tech Research \& Development (863) Program (No. 2013AA020102) and the National Science and Technology Major Project (No. 2012ZX10002004).

\section{Disclosure Statement}

The authors declare that they have no competing interests.

\section{References}

1 Campard D, Lysy PA, Najimi M, Sokal EM: Native umbilical cord matrix stem cells express hepatic markers and differentiate into hepatocyte-like cells. Gastroenterology 2008;134:833-848.

2 Sabin K, Kikyo N: Microvesicles as mediators of tissue regeneration. Transl Res 2013;163:286-295.

3 Cao H, Yang J, Yu J, Pan Q, Li J, Zhou P, Li Y, Pan X, Wang Y, Li L: Therapeutic potential of transplanted placental mesenchymal stem cells in treating chinese miniature pigs with acute liver failure. BMC Med 2012;10:56.

4 Ogawa S, Miyagawa S: Potentials of regenerative medicine for liver disease. Surg Today 2009;39:10191025. 


\section{Cellular Physiology Cell Physiol Biochem 2015;35:2299-2308

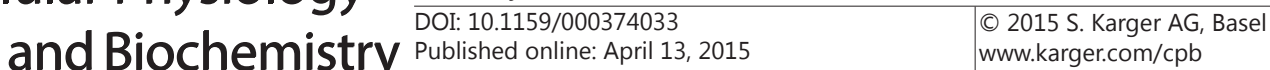 \\ Yu et al.: GFP Labeling Human Mesenchymal Stem Cells}

5 Bouchez G, Sensebe L, Vourc'h P, Garreau L, Bodard S, Rico A, Guilloteau D, Charbord P, Besnard JC, Chalon S: Partial recovery of dopaminergic pathway after graft of adult mesenchymal stem cells in a rat model of parkinson's disease. Neurochem Int 2008;52:1332-1342.

6 Schmidtke-Schrezenmeier G, Urban M, Musyanovych A, Mailander V, Rojewski M, Fekete N, Menard C, Deak E, Tarte K, Rasche V, Landfester K, Schrezenmeier H: Labeling of mesenchymal stromal cells with iron oxide-poly(l-lactide) nanoparticles for magnetic resonance imaging: Uptake, persistence, effects on cellular function and magnetic resonance imaging properties. Cytotherapy 2011;13:962-975.

7 Wolfs E, Struys T, Notelaers T, Roberts SJ, Sohni A, Bormans G, Van Laere K, Luyten FP, Gheysens O, Lambrichts I, Verfaillie CM, Deroose CM: 18f-fdg labeling of mesenchymal stem cells and multipotent adult progenitor cells for pet imaging: Effects on ultrastructure and differentiation capacity. J Nucl Med 2013;54:447-454.

8 Bensidhoum M, Chapel A, Francois S, Demarquay C, Mazurier C, Fouillard L, Bouchet S, Bertho JM, Gourmelon P, Aigueperse J, Charbord P, Gorin NC, Thierry D, Lopez M: Homing of in vitro expanded stro1- or stro-1+ human mesenchymal stem cells into the nod/scid mouse and their role in supporting human cd34 cell engraftment. Blood 2004;103:3313-3319.

9 Karnoub AE, Dash AB, Vo AP, Sullivan A, Brooks MW, Bell GW, Richardson AL, Polyak K, Tubo R, Weinberg RA: Mesenchymal stem cells within tumour stroma promote breast cancer metastasis. Nature 2007;449:557-563.

10 Park YH, Yun JI, Han NR, Park HJ, Ahn JY, Kim C, Choi JH, Lee E, Lim JM, Lee ST: Mass production of earlystage bone-marrow-derived mesenchymal stem cells of rat using gelatin-coated matrix. BioMed research international 2013;2013:347618.

11 Coyne TM, Marcus AJ, Woodbury D, Black IB: Marrow stromal cells transplanted to the adult brain are rejected by an inflammatory response and transfer donor labels to host neurons and glia. Stem Cells 2006;24:2483-2492.

12 Walczak P, Kedziorek DA, Gilad AA, Lin S, Bulte JW: Instant mr labeling of stem cells using magnetoelectroporation. Magn Reson Med 2005;54:769-774.

13 Cromer Berman SM, Walczak P, Bulte JW: Tracking stem cells using magnetic nanoparticles. Wiley Interdiscip Rev Nanomed Nanobiotechnol 2011;3:343-355.

14 Chen IY, Greve JM, Gheysens O, Willmann JK, Rodriguez-Porcel M, Chu P, Sheikh AY, Faranesh AZ, Paulmurugan R, Yang PC, Wu JC, Gambhir SS: Comparison of optical bioluminescence reporter gene and superparamagnetic iron oxide $\mathrm{mr}$ contrast agent as cell markers for noninvasive imaging of cardiac cell transplantation. Mol Imaging Biol 2009;11:178-187.

15 Cheng L, Fu J, Tsukamoto A, Hawley RG: Use of green fluorescent protein variants to monitor gene transfer and expression in mammalian cells. Nat Biotechnol 1996;14:606-609.

16 Leiker M, Suzuki G, Iyer VS, Canty JM Jr, Lee T: Assessment of a nuclear affinity labeling method for tracking implanted mesenchymal stem cells. Cell Transplant 2008;17:911-922.

17 McMahon J, Conroy S, Lyons M, Greiser U, O'shea C, Strappe P, Howard L, Murphy M, Barry F, O'brien T: Gene transfer into rat mesenchymal stem cells: A comparative study of viral and nonviral vectors. Stem cells and development 2006;15:87-96.

18 Van Damme A, Thorrez L, Ma L, Vandenburgh H, Eyckmans J, Dell'Accio F, De Bari C, Luyten F, Lillicrap D, Collen D, VandenDriessche T, Chuah MK: Efficient lentiviral transduction and improved engraftment of human bone marrow mesenchymal cells. Stem Cells 2006;24:896-907. 